\title{
Necesidades hídricas del frijol (Phaseolus vulgaris L.) INTA rojo en condiciones del trópico húmedo de Nicaragua
}

\section{Hydric needs of beans (Phaseolus vulgaris L.) INTA red in conditions of the humid tropic of Nicaragua}

\author{
Juan Asdrúbal Flores-Pacheco ${ }^{1,2}$, Wilfredo Josiel Lazo Sánchez ${ }^{1}$, Jasvin Joel Méndez Sevilla ${ }^{1}$ \\ ${ }^{1}$ Facultad de Recursos Naturales y Medio Ambiente (FARENA), ORCID: https://orcid.org/0000-0001-6553-7202, asdrubal.flores@do.bicu \\ edu.ni, jossiellazo@yahoo.com, sevillajasvin@gmail.com \\ ${ }^{2}$ Dirección de Investigación y Postgrado (DIP) \\ Bluefields Indian \& Caribbean University - BICU, Apartado postal 88, Avenida Universitaria, Bluefields, Nicaragua \\ Autor para correspondencia: asdrubal.flores@do.bicu.edu.ni / + (505) 8369-726
}

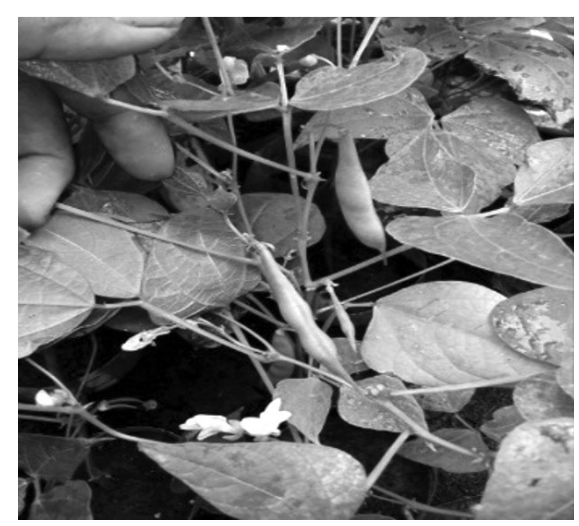

\section{RESUMEN}

El cambio climático ha venido alterando las condiciones agroecológicas en todo el mundo. América Latina es una de las regiones más afectadas, siendo Nicaragua, Honduras y Haití los países más vulnerables. Parte de estos efectos se notan en las variaciones de los regímenes de lluvias al punto de tocar extremos como sequias e inundaciones en zonas de clima moderado. El riesgo se acrecienta al saber que el $90 \%$ de los cultivos de esta región depende del régimen de lluvias. Como alternativa se han comenzado a evaluar y utilizar ampliamente variedades resistentes a la sequía, una de ellas es la variedad de frijol (Phaseolus vulgaris L.) INTA Rojo (EAP 951077). El objetivo de esta investigación es evaluar el comportamiento fenológico y productivo de esta leguminosa en condiciones de estrés hídrico. También se determinará la cantidad mínima y máxima de agua con la que la planta no presente en pérdida productiva por medio de correlaciones de variables. La investigación se realizó en el municipio de Bocana de Paiwas, en el Caribe de Nicaragua en periodo de primera del ciclo productivo 2018. Se registró una precipitación promedio de $60 \mathrm{~mm}$ mensuales. Este estudio es de carácter experimental y dirigido a cuantificar el efecto de las alteraciones fenológicas y productivas en la planta de $P$. vulgaris por el estrés hídrico. Se empleó el Análisis de Varianza y Diferencia Mínima Significativa con un $\alpha=0.05$ para el análisis estadístico de los datos registrados. El tratamiento cinco $\left(\mathrm{T}_{5}\right)$ es el que permitió el desarrollo óptimo de las características fenológicas y productivas $P$. vulgaris. Este cultivar de frijol requiere un mínimo del $80 \%$ y un máximo

\section{ABSTRACT}

Climate change has been altering agroecological conditions throughout the world. Latin America is one of the most affected regions, with Nicaragua, Honduras and Haiti being the most vulnerable countries. Part of these effects are noted in the variations of rainfall regimes to the point of touching extremes such as drought and flooding in areas of moderate climate. The risk increases when you know that $90 \%$ of the crops in this region depend on the rainfall regime. As an alternative, drought-resistant varieties have been widely evaluated and used, one of which is the variety of beans (Phaseolus vulgaris L.) INTA Red (EAP 9510-77). The objective of this research is to evaluate the phenological and productive behavior of this legume in conditions of water stress. It will also determine the minimum and maximum amount of water with which the plant does not enter productive loss by means of variable correlations. The investigation was conducted in the municipality of Bocana de Paiwas, in the Caribbean of Nicaragua during the first period of the productive cycle 2018. An average rainfall of $60 \mathrm{~mm}$ per month was registered. This study is of an experimental nature and aimed at quantifying the effect of the phenological and productive alterations in the plant of $P$. vulgaris due to water stress. The Analysis of Variance and Minimum Significant Difference was used with $\alpha=0.05$ for the statistical analysis of the recorded data. Treatment five $\left(\mathrm{T}_{5}\right)$ is the one that allowed the optimal development of the phenological and productive characteristics $P$. vulgaris. This bean cultivar requires a minimum of $80 \%$ and a maximum of $120 \%$ of the daily amount

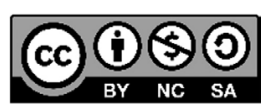

Los artículos de la revista La Calera de la Universidad Nacional Agraria, Nicaragua, se comparten bajo términos de la licencia Creative Commons: Reconocimiento, No Comercial, Compartir Igual. Las autorizaciones adicionales a las aquí delimitadas se pueden obtener en el correo edgardo.jimenez@ci.una.edu.ni

(C) Copyright 2019. Universidad Nacional Agraria 
$120 \%$ de la cantidad diaria de evapotranspiración para no entrar en pérdida productiva por estrés hídrico. Partiendo del dato anterior se identifica que el cultivar del frijol es altamente (0.95) sensible a las variaciones de humedad en el suelo. Además, no presenta características de adaptación a condiciones de sequía y/o estrés hídrico por carencia de agua. Se requieren más investigaciones en condiciones controladas donde se pueda monitorear variables ambientales y atmosféricas. También deben integrarse otras variedades, entre ellas las criollas de uso común.

Palabras clave: índice de productividad, sequia, variedad tolerante, adaptación climática. of evapotranspiration to avoid losing production due to water stress. From the previous data it is identified that the bean cultivar is highly $(0.95)$ sensitive to variations in soil moisture. In addition, it does not have characteristics of adaptation to drought conditions and / or water stress due to lack of water. More research is required in controlled conditions where environmental and atmospheric variables can be monitored. Other varieties must also be integrated, among them the local of common use.

Keywords: Productivity index, drought, tolerant variety, climatic adaptation.

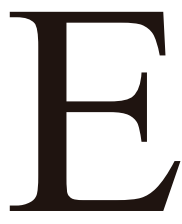

1 presente estudio tuvo como fin obtener el potencial del rendimiento del cultivo de frijol (Phaseolus vulgaris L.) variedad INTA Rojo (EAP 9510-77) de acuerdo a distintos niveles de estrés hídrico, esto a fin de comparar los resultados con lo reportado por el Instituto Nicaragüense de Tecnología Agropecuaria (INTA) (INTA, 2009). El frijol es un cultivo que no requiere grandes volúmenes de agua durante su ciclo vegetativo, la demanda de agua dependió en su fase de desarrollo, siendo el cultivo exigente en la fase de germinación y muy exigente en la fase de diferenciación floral, fructificación y llenado del grano (IN A, 2009).

En un contexto de clima variable, la capacidad de transformar la agricultura para alimentar a una población creciente sin perjudicar la base de recursos naturales no sólo permitió alcanzar los objetivos de seguridad alimentaria, sino que también ayuda a mitigar los efectos negativos del cambio climático. Una agricultura más productiva y resistente requerirá una mejor gestión de los recursos naturales, como la tierra, el agua, el suelo y los recursos genéticos, a través de buenas prácticas como la agricultura de conservación, el control integrado de plagas, la agroforestería y las dietas sostenibles, para identificar genotipos con resistencia múltiple a sequía y enfermedades, el mejoramiento genético se ha basado en métodos tradicionales que requieren de la evaluación de un alto número de genotipos, para incrementar las probabilidades de seleccionar los que expresen un comportamiento superior (Meriño et al. 2015)

En la última década, los enfoques utilizados por los programas de mejoramiento han sido enfatizados en la generación de cultivares con una base genética más amplia y mayor adaptación regional. Para ello, fuentes diversas de germoplasma de las razas andinas y mesoamericanas se emplean en los programas más relevantes en la región de Centroamérica y el Caribe (Correia et al. 2014). Actualmente, algunas variedades y germoplasma mejoradas poseen una base genética adecuada que les confiere mayor adaptación y potencial de rendimiento, así como mejor resistencia a enfermedades y factores abióticos, que las variedades criollas. Lamentablemente, los beneficios derivados del empleo de variedades mejoradas están frecuentemente limitados por sistemas deficientes de diseminación y el reducido acceso de los agricultores a semilla de alta calidad. En otros casos, las variedades mejoradas no son adoptadas debido a su inferior calidad de grano con respecto a las criollas, o la falta de adaptación a los sistemas de producción de bajos insumos usados por los pequeños productores (Acosta-Diaz, Kohashi-Shibata y Acosta-Gallegos, 1997).

Como salida a esta problemática se ha establecido un experimento para determinar el efecto del estrés hídrico en la variedad de frijol INTA Rojo (EAP 9510-77). Se estableció en el municipio de Bocana de Paiwas, entre los meses de abril a julio del año 2018 correspondiente al periodo de primera en el ciclo productivo agrícola nicaragüense. Esta zona está caracterizada por ser parte del Trópico Húmedo de Nicaragua de acuerdo a la caracterización climática del Instituto Nicaragüense de Estudios Territoriales (INETER, 2015).

\section{MATERIALES Y MÉTODOS}

Localización del estudio. Se realizó en la comunidad El Toro, ubicado en el municipio de Bocana de Paiwas en la Región Autónoma Costa Caribe Sur (Figura 1), durante los meses de abril a julio del año 2018 coincidente con la época de primera del ciclo agrícola nacional. Se eligió la finca San Isidro por medio de consultas a productores interesados en mejorar la productividad de sus cultivos y con la disponibilidad de facilitar el espacio para la realización del experimento. $\mathrm{La}$ finca cuenta con un área total de cinco hectáreas (ha) de las cuales se destinan 1.8 ha para el cultivo de frijol. La parcela se seleccionó para el experimento basados en la producción previa de este cultivo en el área. Tomando como área útil para el experimento de $385 \mathrm{~m}^{2}$. Esta fue cercada perimetralmente con láminas de zinc como medida de protección contra plagas y personas ajenas al equipo de investigación. Se colocó un sistema móvil de plástico negro para cubrir las plantas en momentos de lluvia y por las noches garantizando que no se adicionara más agua que la calculada para cada tratamiento. Durante el día y en momentos en que no se registró precipitación este sistema móvil fue retirado para evitar afectaciones a las tasas fotosintéticas del cultivo. 
Las variables ambientales de temperatura $\left({ }^{\circ} \mathrm{C}\right)$ y humedad relativa (\%) se registraron a las 8:00 am y 2:00 pm de la hora local según lo indicado por la publicación de Benavides y Díaz (1970) para cálculo de la Evapotranspiración potencial entre los $15^{\circ}$ Norte y $15^{\circ}$ Sur. Los datos fueron consultados en el sitio web oficial del Instituto Nacional de Estudios Territoriales (INETER) durante el periodo de ejecución del estudio. Sin embargo, por carencia de equipos especializados estas variables no se pudieron controlar y medir directamente en el sitio del experimento.

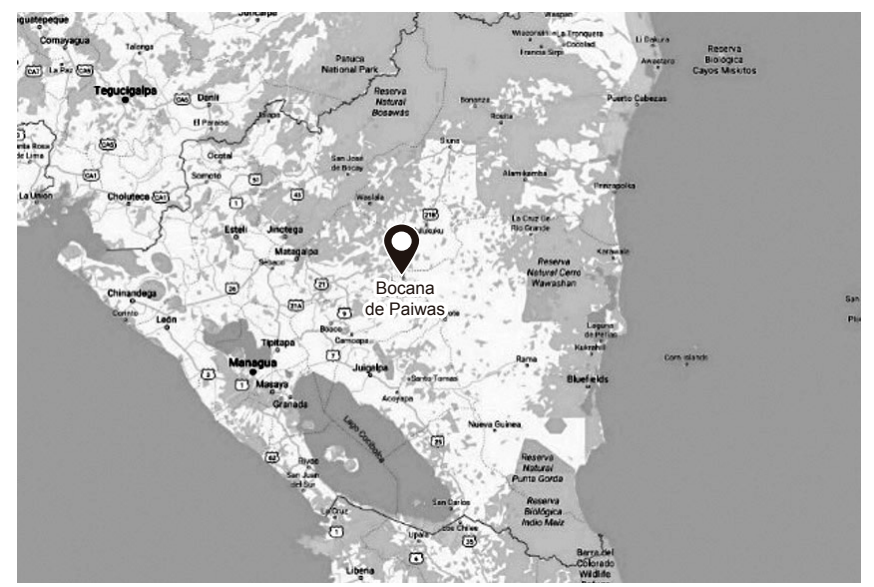

Figura 1. Ubicación de la comunidad El Toro, Bocana de Paiwas, Región Autónoma Costa Caribe Sur, Nicaragua.

Establecimiento del experimento. El experimento se estableció en una parcela de 35 metros de longitud por 11 metros de ancho para un área útil de $385 \mathrm{~m}^{2}$ (Figura. 2). En cada tratamiento se establecieron 50 réplicas de las cuales se muestrearon las 20 centrales descartando 15 en cada extremo de la columna por el efecto de borde. Las plantas se establecieron en maceteras elaborada a base de fibras vegetales: $80 \%$ de fibra de madera y en un $20 \%$ de turba de $1000 \mathrm{ml}$ (equivalente a $0.001 \mathrm{~m}^{3}$ ) de capacidad con sustrato mejorado consistente en un compuesto de $50 \%$ de tierra, $30 \%$ de arena de río y $20 \%$ de compost obteniendo una textura franco-arenosa (Flores-Pacheco, Flores-Pacheco, Murillo, Oporta, y Alemán 2016a).

Previo a la siembra se realizó la prueba de germinación que consistió en la colocación de 100 semillas en un plato de plástico con papel absorbente humedecidos (aprox. $70 \%$ de humedad) por 72 horas continuas en condiciones ambientales (Flores-Pacheco, Godoy, Rostrán y Bárcenas, 2015). Al transcurrir este tiempo se contabilizó la cantidad de semillas germinadas, para este caso la germinación alcanzó $87 \%$, en base a ello se colocaron cinco semillas por macetera para garantizar una planta por envase. En el caso de germinación múltiple, se eliminaron (ralearon) las plantas excedentes dejando la más vigorosa. Las maceteras fueron colocadas a distancias de 0.10 metros entre ellas y 2 metros entre calles, esto se hizo para contar con mayor control de las variables monitoreadas por cada repetición y tratamiento (Flores-Pacheco, Ramírez-James, Gutiérrez-Rugama, Flores-Pacheco y Alemán, 2016b).

Se emplearon 50 réplicas por cada uno de los siete tratamientos (350 en total) para asegurar cantidad suficiente de plantas en el caso de muerte por causa natural, por plagas, enfermedades y para reducir el efecto de borde muestreando las que se encuentran en el centro (Monzón y Monzón-Paiva, 1992).

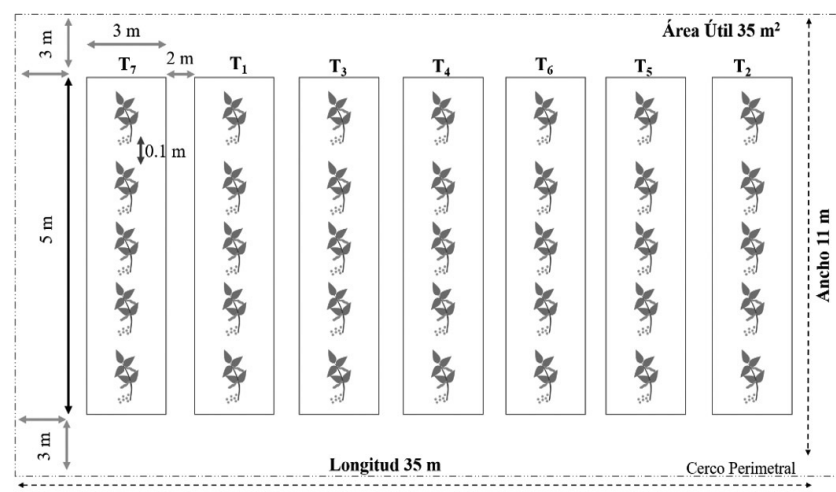

Figura 2. Esquema de la distribución de los tratamientos en el campo.

El estudio fue de tipo experimental, con enfoque cuantitativo, debido a la manipulación de variables especificas a fin de obtener datos para el análisis del comportamiento fenológico y productivo de las plantas frijol sometido a distintos niveles de estrés hídrico. Se empleó un diseño experimental Aleatorio Simple de repeticiones balanceadas con 95\% de confiabilidad (García-Pérez, 2010). La medición de variables de este estudio fue de corte transversal. Desarrollándose la investigación en un periodo de 120 días, (4 meses) de campo (ciclo productivo) y 90 días (3 meses) para el análisis de datos y presentación de resultados, para un ciclo aproximado de siete meses.

Unidad experimental. Se consideró como unidad experimental cada una de las plantas de frijol y se empleó el Muestreo Aleatorio Simple (MAS) con frecuencia semanal para las variables fenológicas y única para las variables productivas. En base a los datos obtenidos del portal web de INETER se calculó la Evapotranspiración Potencial Diaria con lo cual se determinó la cantidad de agua a emplear en cada tratamiento. El agua fue medida con un recipiente milimetrado con el cual se vertió directamente en la base del tallo a una distancia que oscilaba entre los $5-10 \mathrm{~cm}$. Las aplicaciones se realizaron diariamente entre el día 0 al 60 (abril y mayo de 2018) aproximadamente entre las 3:00 pm y 4:00 pm. Para evitar la incorporación de agua adicional a cada tratamiento en forma 
de lluvia y/o rocío, se colocó el sistema de plástico portable que cubría la totalidad del área del experimento.

Cuadro 1. Tratamientos y repeticiones

\begin{tabular}{clc}
\hline Código & \multicolumn{1}{c}{ Tratamientos evaluados } & Repeticiones \\
\hline $\mathrm{T}_{1}$ & $20 \%$ de la evapotranspiración & 50 \\
$\mathrm{~T}_{2}$ & $50 \%$ de la evapotranspiración & 50 \\
$\mathrm{~T}_{3}$ & $80 \%$ de la evapotranspiración & 50 \\
$\mathrm{~T}_{4}$ & $100 \%$ de la evapotranspiración (Testigo) & 50 \\
$\mathrm{~T}_{5}$ & $120 \%$ de la evapotranspiración & 50 \\
$\mathrm{~T}_{6}$ & $150 \%$ de la evapotranspiración & 50 \\
$\mathrm{~T}_{7}$ & $180 \%$ de la evapotranspiración & 50 \\
\hline Total & & 350 \\
\hline
\end{tabular}

Cálculo de la evapotranspiración potencial diaria. Es una fórmula del Método de García y López (Benavides y Díaz, 1970) adaptada al trópico que permite estimar la evapotranspiración potencial diaria, entre latitudes $15^{\circ}$ Norte y $15^{\circ}$ Sur. Desarrollada a partir de la correlación de los datos de temperatura y déficit de saturación con los de la evapotranspiración potencial medidos en seis estaciones tropicales. La ecuación se presenta a continuación:

$$
E T P=1.21 \times 10^{F t}\left(1-0.01 H R_{D}\right)+0.21 t-2.3 \quad(\text { Ecuación } 1)
$$

Ft es el factor de temperatura el cual se define en la ecuación 2:

$$
F t=\frac{7.45 t}{234.7+t} \quad(\text { Ecuación } 2)
$$

Donde $t$ es la temperatura media mensual del área en grados Celsius $\left({ }^{\circ} \mathrm{C}\right)$ y $\mathrm{HR}_{\mathrm{D}}$ es la Humedad Relativa (\%) diurna que se define como sigue en la siguiente expresión

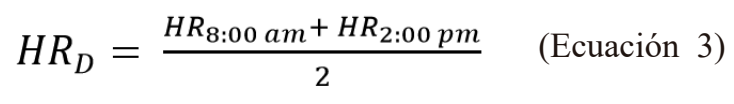

Los datos de temperatura diaria y Humedad Relativa diaria fueron obtenidos del registro en línea del Instituto Nacional de Estudios Territoriales (INETER) (www.ineter.gob. ni). De acuerdo con la formula emplea se utilizaron los valores de la humedad relativa registrados en este sitio web para las 8:00 am y 2:00 pm. Los datos obtenidos alimentaron una hoja de cálculo automatizada en Microsoft Excel ${ }^{\circledR}$ que facilitó la aplicación de las fórmulas descritas anteriormente y la estimación más exacta de la cantidad de agua suministrada a cada planta según el tratamiento evaluado.

Análisis estadístico. Los datos fueron procesados en el programa estadístico SPSS $24.0\left(\right.$ IBM ${ }^{\circledR}$ Statistical SPSS ${ }^{\circledR}$,
2016). Todas las variables cuantitativas fueron analizadas para comprobar la normalidad y homogeneidad de varianzas, bajo este supuesto se aplicaron las pruebas de Coeficiente de variación (CV), Análisis de Varianza (ANOVA) y Diferencia Mínima Significativa (DMS) de Fisher con $\alpha=0.05$, esto con el fin de identificar las diferencias en las variables evaluadas para cada tratamiento.

\section{RESULTADOS Y DISCUSIÓN}

Variables fenológicas del frijol antes el estrés hídrico. $\mathrm{La}$ figura $3 \mathrm{~A}$ y $3 \mathrm{~B}$ representan la altura $(\mathrm{cm})$ y diámetro $(\mathrm{mm})$ de la planta de frijol sometido a distintos niveles de estrés hídrico a lo largo de un ciclo productivo de 120 días en condiciones del trópico húmedo de Nicaragua. Los resultados indican que el tratamiento cinco $\left(\mathrm{T}_{5}=\right.$ suministro del $120 \%$ de evapotranspiración diaria) obtuvo los registros más elevados en altura $\pm 20.57 \mathrm{~cm}(\mathrm{~F}=12.57 ; \mathrm{gl}=6$; $\mathrm{P}$-valor $>0.031)$ y diámetro $\pm 2.40 \mathrm{~mm}(\mathrm{~F}=5.40 ; \mathrm{gl}=6$; P-valor $>0.029)$ frente a los demás tratamientos que presentaron un desarrollo inferior tanto en el segmento inferior como superior de la dosis de agua diaria. Este tratamiento superó estadísticamente por $11.38 \%$ y 19.94\% Coeficiente de variación (CV) respectivamente, más en crecimiento al tratamiento cuatro $\left(\mathrm{T}_{4}=\right.$ suministro del $100 \%$ de evapotranspiración diaria), para esta investigación considerado como el testigo.

El tratamiento seis $\left(\mathrm{T}_{6}=\right.$ suministro del $150 \%$ de evapotranspiración diaria) indica una reducción significativa (P-valor $>0.001)$ en crecimiento (altura y diámetro) respecto al testigo $\left(\mathrm{T}_{4}\right)$ demostrando que este cultivar de $P$. vulgaris es susceptible a estrés hídrico por exceso de agua en el suelo, posiblemente por un problema de hipoxia en el sustrato. Aguilar-Benítez et al. (2012) hacen referencia a la imposibilidad de esta leguminosa a la asimilación de nutrientes en suelos con niveles de humedad que supere la capacidad de campo.

En el caso del cultivo de frijol requiere que el suelo presente un 60\%-70\% de humedad a lo largo del ciclo productivo con énfasis en la fase de crecimiento vegetativo y el inicio de la producción (Meriño et al. 2015). Sin embargo, muchas veces se obvia la retención de agua por el suelo como agua no disponible, la que puede llegar a ser hasta más de un $20 \%$ de la evaporación registrada en el día, eso en función al tipo de suelo (Velarde et al. 2001). Este hecho explica el éxito del T5 ya que en ambos caso la planta cuenta con la abundancia y disponibilidad de agua requerida para sostener sus necesidades metabólicas y nutricionales (Benavides et al. 2002). Para este estudio se descarta el efecto de bajos valores de $\mathrm{pH}$ y salinidad en los suelos por empleo de fertilizantes sintéticos por el hecho de que el estudio se llevó enteramente en recipientes plásticos con suelo natural de tipo franco arcilloso. Sin embargo, debe ser considerados en futuras investigaciones que se recomienda se desarrollen en parcelas productivas sometidas a distintos tipos de manejo agronómico. 

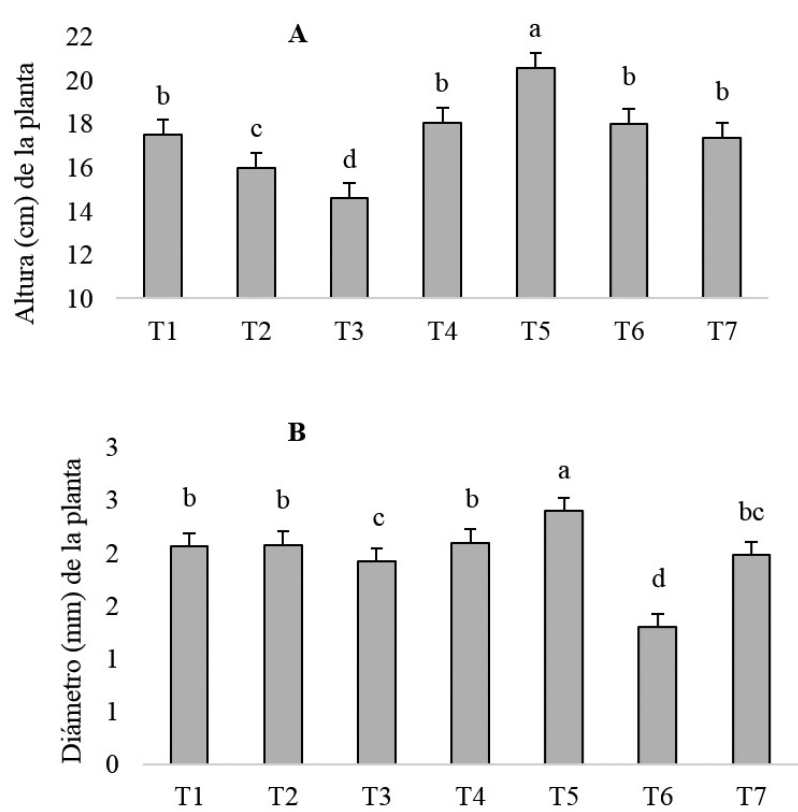

Figura 3. Altura de planata en centímetros A) y diámetro del tallo en milímetro (B) en función de los tratamientos.

En lo relativo al comportamiento de la sección radicular de la planta de frijol (P. vulgaris) la cuadro 2 muestra que los tratamientos que suministran únicamente el $20 \%$,
$50 \%$ y $80 \%\left(\mathrm{~T}_{1}, \mathrm{~T}_{2}\right.$ y $\mathrm{T}_{3}$, respectivamente $)$ de la cantidad de agua evapotranspirada por la planta presentan reducción significativa (P-valor $>0.001)$ respecto al tratamiento testigo $\left(\mathrm{T}_{4}\right)$ variando $(\mathrm{CV}) 23.66 \%$. Esto se mostró en raíces de menor tamaño, de mayor fragilidad al contacto y de menor concentración de agua (Valladares, 2004). Sin embargo, para los tratamientos que adicionan el $120 \%, 150 \%$ y $180 \%$ de la cantidad de agua evapotranspirada por la planta $\left(\mathrm{T}_{5}, \mathrm{~T}_{6} \mathrm{y}\right.$ $\mathrm{T}_{7}$, respectivamente) presentan un incremento significativo (P-valor $>0.001)$ en estas mismas características de la raíz de $P$. vulgaris en el mismo orden de los tratamientos. Para las secciones del tallo y foliar el comportamiento siguió la tendencia ya descrita. Los $\mathrm{T}_{1}, \mathrm{~T}_{2}$ y $\mathrm{T}_{3}$ presentaron reducciones significativ $\mathrm{s}$ en las cantidades de agua registrada en el momento del peso fresco y el peso seco. Debe destacarse que a pesar de que los tratamientos $\mathrm{T}_{5}, \mathrm{~T}_{6} \mathrm{y}_{7}$ presentaron una relación positiva ( $\mathrm{P}$-valor $>0.001 ; \mathrm{CV}=8.06 \%$ ) en este parámetro al realizar comparaciones entre estos es el $\mathrm{T}_{5}$ el más eficiente en el uso del agua. Esto en que no existe diferencia $(\mathrm{P}$-valor $<0.083 ; \mathrm{CV}=1.14 \%)$ entre estos tratamientos, por ello, dará el mismo resultado la adición de $120 \%$ del índice de evatranspiración que cualquier otra cantidad mayor, esto es coincidente con lo reportado por (Moreno, 2009) en experimentos de estrés hídrico desarrollado en hortalizas, gramíneas y leguminosas bajo condiciones controladas.

Cuadro 2. Análisis comparativo de la biomasa por cada sección de la planta en función del tratamiento

\begin{tabular}{|c|c|c|c|c|c|c|c|}
\hline Tratamiento & Sección & Peso $(g)$ & Md & Sig. & Peso $(g)$ & Md & Sig \\
\hline $\mathrm{T}_{1}=20 \%$ de la evapotranspiración & \multirow{7}{*}{ 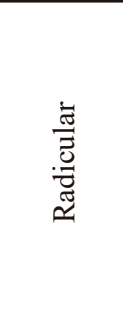 } & \multirow{7}{*}{$\begin{array}{l}\text { 욜 } \\
\text { 莺 }\end{array}$} & 1.30 & $* *$ & \multirow{7}{*}{$\begin{array}{l}8 \\
\text { D } \\
\text { ñ }\end{array}$} & 0.65 & ** \\
\hline $\mathrm{T}_{2}=50 \%$ de la evapotranspiración & & & 1.40 & $* *$ & & 0.70 & $* *$ \\
\hline $\mathrm{T}_{3}=80 \%$ de la evapotranspiración & & & 2.00 & $*$ & & 0.80 & $* *$ \\
\hline $\mathrm{T}_{4}=100 \%$ de la evapotranspiración & & & 2.00 & ns & & 0.99 & ns \\
\hline $\mathrm{T}_{5}=120 \%$ de la evapotranspiración & & & 2.00 & $*$ & & 1.50 & ns \\
\hline $\mathrm{T}_{6}=150 \%$ de la evapotranspiración & & & 2.00 & $* *$ & & 1.37 & ns \\
\hline $\mathrm{T}_{7}=180 \%$ de la evapotranspiración & & & 2.00 & $* *$ & & 1.40 & ns \\
\hline $\mathrm{T}_{1}=20 \%$ de la evapotranspiración & \multirow{7}{*}{$\stackrel{\circ}{\stackrel{\bar{\pi}}{๘}}$} & \multirow{7}{*}{ 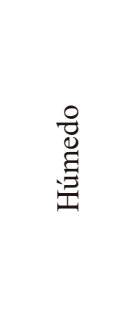 } & 1.00 & ns & \multirow{7}{*}{$\begin{array}{l}\text { ठ } \\
\text { W }\end{array}$} & 0.50 & $* *$ \\
\hline $\mathrm{T}_{2}=50 \%$ de la evapotranspiración & & & 1.00 & ns & & 0.50 & $*$ \\
\hline $\mathrm{T}_{3}=80 \%$ de la evapotranspiración & & & 1,00 & ns & & 0.45 & $*$ \\
\hline $\mathrm{T}_{4}=100 \%$ de la evapotranspiración & & & 1.00 & ** & & 0.49 & ns \\
\hline $\mathrm{T}_{5}=120 \%$ de la evapotranspiración & & & 2.07 & $* *$ & & 1.00 & $* *$ \\
\hline $\mathrm{T}_{6}=150 \%$ de la evapotranspiración & & & 2.00 & $* *$ & & 1.47 & $* *$ \\
\hline $\mathrm{T}_{7}=180 \%$ de la evapotranspiración & & & 3.00 & $* *$ & & 0.79 & $* *$ \\
\hline $\mathrm{T}_{1}=20 \%$ de la evapotranspiración & \multirow{7}{*}{ 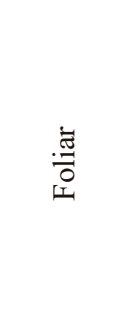 } & \multirow{7}{*}{$\begin{array}{l}\text { 욜 } \\
\text { 吾 }\end{array}$} & 2.00 & ns & \multirow{7}{*}{$\begin{array}{l}8 \\
\text { D } \\
\text { W }\end{array}$} & 1.10 & $* *$ \\
\hline $\mathrm{T}_{2}=50 \%$ de la evapotranspiración & & & 2.50 & ns & & 1.25 & $*$ \\
\hline $\mathrm{T}_{3}=80 \%$ de la evapotranspiración & & & 2.00 & ns & & 1.03 & $*$ \\
\hline $\mathrm{T}_{4}=100 \%$ de la evapotranspiración & & & 2.00 & $* *$ & & 1.11 & ns \\
\hline $\mathrm{T}_{5}=120 \%$ de la evapotranspiración & & & 3.29 & $* *$ & & 1.50 & $* *$ \\
\hline $\mathrm{T}_{6}=150 \%$ de la evapotranspiración & & & 4.00 & $* *$ & & 2.80 & $* *$ \\
\hline $\mathrm{T}_{7}=180 \%$ de la evapotranspiración & & & 4.00 & $* *$ & & 1.90 & $* *$ \\
\hline
\end{tabular}

$\mathrm{Md}=$ Media aritmética. Sig. = significancia estadística al $0.05^{*}$ o al $0.001^{* *}$. Diferencia Mínima Significativa $(\mathrm{DMS}=0.95$ 
Comportamiento de las variables productivas del frijol antes el estrés hídrico. En la figura 4A en la que se muestra el número de flores por planta queda evidenciada la diferencia entre los tratamientos evaluados, donde el $\mathrm{T}_{5}$ presenta mayor número con $\pm 7.22(\mathrm{~F}=12.51 ; \mathrm{gl}=6$; $\mathrm{P}$-valor $>0.000)$ por planta. Esto se debe a la menor cantidad de agua empleada en este tratamiento. Por su parte en la figura 4B se muestra la variable número de vainas por planta logrando observar que existe un incremento significat vo del $\mathrm{T}_{5}$ con \pm 5.18 vainas por planta $(\mathrm{F}=17.17$; gl=6; P-valor $>0.017)$, con lo que respecta a los demás tratamientos evaluados. La producción de flores y vainas se mostraron más susceptible en los tratamientos $\mathrm{T}_{1}, \mathrm{~T}_{2}, \mathrm{~T}_{3} \mathrm{y} \mathrm{T}_{4}$.

Según Acosta-Díaz, Kohashi-Shibata y Acosta-Gallegos (1997), cuando las precipitaciones están por debajo de las necesidades del cultivo los rendimientos disminuyen drásticamente, fundamentalmente sí coinciden con la fl ración y el llenado de las vainas del cultivo, es decir, que la precipitación acumulada durante la etapa reproductiva es determinante para el rendimiento de frijol (Meriño et al. 2015). Estos resultados coinciden con los referidos por Domínguez-Suárez et al. (2016) cuando encontraron respuestas similares al trabajar con 64 líneas de frijol común en dos condiciones de humedad del suelo. Otros autores como Fabre et al. (2011) refieren que en dependencia de la duración del período de sequía y su magnitud, esta puede causar pérdidas en el rendimiento de $20 \%$ a $100 \%$ en los campos del frijol.
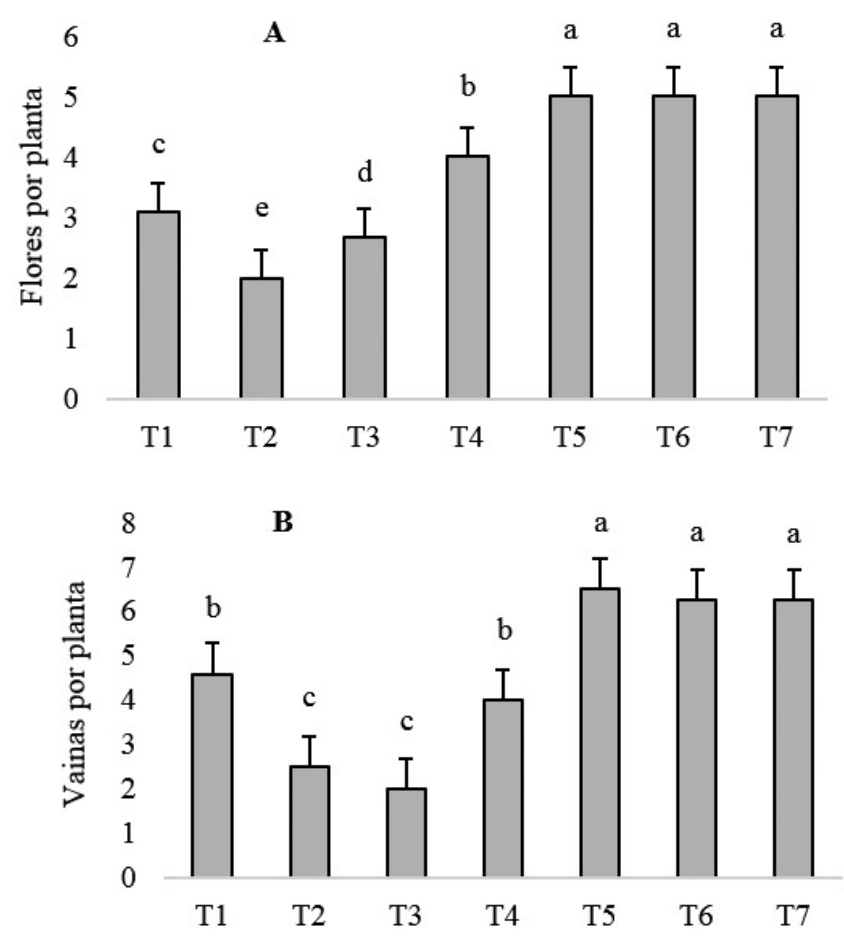

Figura 4. Variables productivas. A) Número de flores por planta B) Número de vainas por planta.
Relación del índice productivo con la cantidad de agua requerida por la planta. Se registraron las variables morfométricas de la vaina. Tanto en la figura 5A para la longitud de la vaina $\pm 10.90 \mathrm{~cm}(\mathrm{~F}=3.66 ; \mathrm{gl}=6$; $\mathrm{P}$-valor $>0.02)$ como en la figura $5 \mathrm{~B}$ para el diámetro de la vaina $\pm 5.60 \mathrm{~mm}(\mathrm{~F}=3.89$; $\mathrm{gl}=6$; P-valor $>0.01)$ el tratamiento cinco $\left(\mathrm{T}_{5}\right)$ obtuvo los registros más elevados entre los siete niveles de estrés hídrico a los cuales se está probando la adaptabilidad de esta leguminosa coincidente con Mivilla, González, y Marulanda (1986). El índice productivo de la variedad mostró respuestas diferentes entre los niveles de estrés hídrico, se pueden considerar con una correlación de 0.95 como las más adecuada a las condiciones que se establecieron en el lugar de los ensayos el tratamiento cinco. Este combina un índice productivo alto y un mayor período desde la floración hasta la madurez, lo que favorece un período amplio para la formación de órganos reproductivos (Domínguez-Suárez et al. 2016).
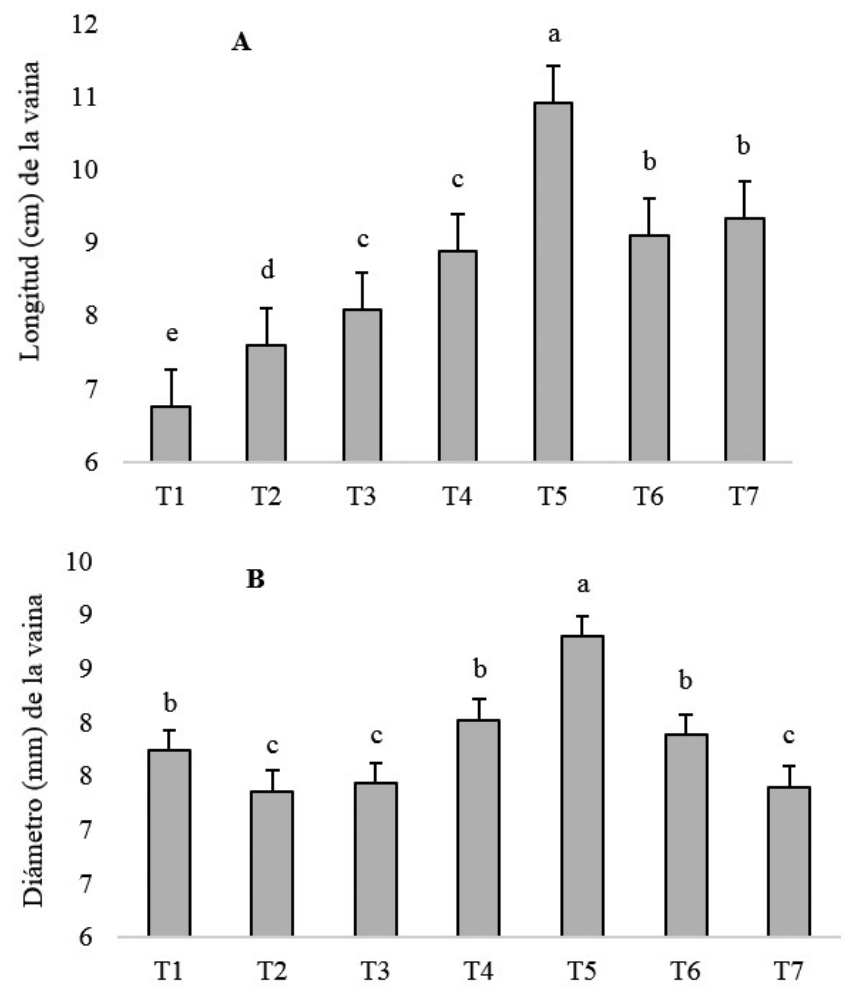

Figura 5. Variables productivas. A) Longitud $(\mathrm{cm})$ y B) Diámetro $(\mathrm{mm})$ de la vaina en función del tratamiento.

Probablemente la pérdida de rendimientos en estos indicadores de productividad estudiados obedezca a la limitada actividad fotosintética y a una menor absorción de nutrientes por la reducida movilidad de iones en el suelo y toma de agua por las raíces (Medrano y Flexas, 2003). Los resultados alcanzados en este trabajo evidenciaron variabilidad entre niveles de estrés hídrico en cuanto a la reducción 
del rendimiento, lo que concuerda con lo reportado por otros autores (Acosta-Diaz, Kohashi-Shibata y Acosta-Gallegos, 1998; Domínguez-Suárez et al. 2016; Mivilla, González y Marulanda, 1986; Prado, Jerez, Nápoles, Sosa, Maceo y Cordoví, 2016; Tosquy-Valle, López-Salinas, Francisco-Nicolás, Alberto y Bernardo, 2014). En varios estudios se ha reiterado el efecto deletéreo de la sequía sobre el crecimiento, el rendimiento y la nutrición mineral de las plantas (Meriño et al. 2015).
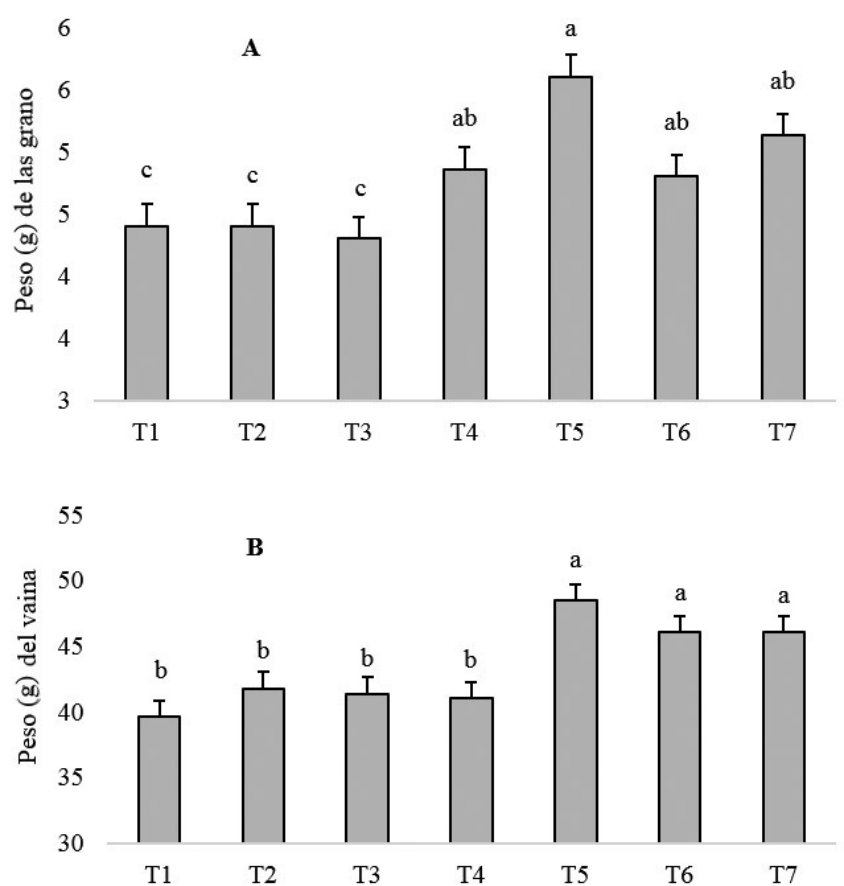

Figura 6. Variables productivas A) Peso (g) de la vaina - B) Peso (g) del grano de la planta en función del tratamiento.

De acuerdo con Allen, Pereira, Raes, y Smith, (2006) la falta de agua durante las etapas de prefloración, formación y llenado de vainas afecta seriamente el rendimiento. El análisis de varianza y comparación de medias al rendi- miento obtenido mostró que existen diferencias significat vas entre las variables Peso del grano \pm 5.60 gr $(\mathrm{F}=26.10$; $\mathrm{gl}=6$; P-valor $>0.000 ; \mathrm{CV}: 8 \%$ ) y Peso de la vaina $\pm 48.46 \mathrm{gr}$ $(\mathrm{F}=10.05 ; \mathrm{gl}=6$; $\mathrm{P}$-valor $>0.000 ; \mathrm{CV}=10 \%)$ a favor del tratamiento cinco $\left(\mathrm{T}_{5}\right)$ que suministra a la planta el $120 \%$ del agua evapotranspirada diariamente. Lo que indica la presencia de una baja adaptabilidad de la variedad a las condiciones de sequía ya que se redujo significativamente su rendimiento en la condición de estrés hídrico por carencia de agua.

La reducción del rendimiento alcanzado en las condiciones experimentales de sequía concuerda con los obtenidos por Arellano-Arciniega, Osuna-Ceja, Martínez-Gamiño y Reyes-Muro (2015) en el cultivo de 10 genotipos en diferentes condiciones de siembra y riego. La reducción de rendimiento por falta o exceso de humedad fue evidente en todos los tratamientos evaluados. La mayoría de los tratamientos con estrés por exceso de agua presentaron moderada tolerancia al estrés hídrico, debido a que obtuvieron índices de producción superior a uno. Los resultados de índices de correlación para los tratamientos con déficit y exceso de agua están entre 0.70 y 0.95 .

\section{CONCLUSIONES}

El tratamiento cinco $\left(\mathrm{T}_{5}\right)$ es el que permitió el desarrollo óptimo de las características fenológicas (altura, diámetro, número de ramas y hojas) y productivas (número de flores, vainas, granos por vaina, biomasa y morfometría del grano) del frijol (Phaseolus vulgaris L) variedad INTA Rojo (EAP 9510-77) de acuerdo con las características descritas por el Instituto Nicaragüense de Tecnología Agropecuaria. Este cultivar de frijol requiere un mínimo del $80 \%$ y un máximo $120 \%$ de la cantidad diaria de evapotranspiración para no tener pérdidas productivas por estrés hídrico.

Partiendo del dato anterior se identifica que el cultivar del frijol INTA Rojo (EAP 9510-77) es altamente (0.95) susceptible a las variaciones de humedad en el suelo y presenta escasas características de resistencia a condiciones de sequía y/o estrés hídrico por carencia de agua.

\section{REFERENCIAS BIBLIOGRÁFICAS}

Acosta-Díaz, E., Kohashi-Shibata, J., y Acosta-Gallegos, J. A. (1997). Rendimiento y sus componentes en frijol bajo condiciones de sequía. Agricultura Técnica En México, 23(2), 139-151.

Aguilar-Benítez, G., Peña-Valdivia, C. B., García-Nava, J. R., Ramírez-Vallejo, P., Benedicto-Valdés, S. G., y Molina-Galán, J. D. (2012). Rendimiento d frijol (Phaseolus vulgaris L.) en relación con la concentración de vermicompost y déficit de humedad en el sustrato. Agrociencia, 46(1), 37-50. Retrieved from http://www.scielo.org.mx/scielo. php?script $=$ sci arttext\&pid $=$ S1405-31952012000100004

Allen G., R., Pereira, L. S., Raes, D., y Smith, M. (2006). Evapotranspiración del cultivo: Guias para la determinación de los requerimientos de agua de los cultivos. FAO: Estudios FAO Riego y Drenaje 56, 297. https://doi.org/10.1590/1983-40632015v4529143

Arellano-Arciniega, S., Osuna-Ceja, E. S., Martínez-Gamiño, M. A., y Reyes-Muro, L. (2015). Rendimiento de frijol fertilizado con estiércol bovino en condiciones de secano. Revista Fitotecnia Mexicana, 38(3), 313-318. 
Benavides, A., Ramirez, H., Robledo, V., Maiti, R., Cronejo, E., Hernández, J., Fuentes, L. (2002). Ecofisiología y Bioquímica del Estrés en Plantas. (A. B. Mendoza, Ed.) $\left(2^{\circ}\right)$. Mexico. Retrieved from http://www.librosagronomicos. blogspot.mx/

Benavides, J. G., y Díaz, J. L. (1970). Fórmula para el cálculo de la evapotranspiración potencial adaptada al tropico $\left(15^{\circ} \mathrm{N}-15^{\circ} \mathrm{S}\right)$. Agronomía Tropical, 20(5), 335-345. Retrieved from http://sian.inia.gob.ve/repositorio/revistas_ci/ Agronomia Tropical/at2005/arti/garcia_j.htm

Correia, B., Pintó-Marijuan, M., Neves, L., Brossa, R., Dias, M. C., Costa, A., Pinto, G. (2014). Water stress and recovery in the performance of two Eucalyptus globulus clones: Physiological and biochemical profiles. Physiologia Plantarum, 150(4), 580-592. https://doi.org/10.1111/ppl.12110

Domínguez-Suárez, A., Martínez-Dávalos, Y., Pérez-Hernández, Y., Fuentes-Alfonso, L., Darias-Rodríguez, R., Casti1lo, M. S., Castillo, D. S. (2016). Comportamiento de variedades cubanas y venezolanas de frijol común, cultivados en condiciones de sequía. Revista Ciencia UNEMI, 9(20), 68-75.

Fabre, T. B., Secada, Y., Chaveco, O., Boudet, A., Gómez, Y., Meriño, Y., Tornes, N. (2011). Respuesta a la sequía de genotipos de fríjol común utilizando diferentes índices de selección. Cagricola.Uclv.Edu.Cu, 38(4), 69-73. Retrieved from http://cagricola.uclv.edu.cu/descargas/pdf/V38-Numero_4/cag134111824.pdf

Flores-Pacheco, J. A., Godoy, S., Rostrán, J., y Bárcenas, M. (2015). Efecto de la poda de guías y dos tipos de fertilización en la producción de Melón (Cucumis melo). Revista Universitas, Universidad Nacional Autónoma de Nicaragua-León, Nicaragua, 6 (ISSN 2311-6072), 186-198.

Flores-Pacheco, J. A., Flores-Pacheco, C. J., Murillo, Y., Oporta, R., y Alemán, Y. (2016a). Evaluación de la producción de tomate (Lycopersicum sculemtun) y chiltoma (Capsicum annuum) con diferentes sustratos hidropónicos. Nexo Revista Cientifica, Universidad Nacional de Ingeniería (UNI), Nicaragua, (ISSN 1998-8850.).

Flores-Pacheco, J. A., Ramírez-James, M., Gutiérrez-Rugama, A., Flores-Pacheco, C. J., y Alemán, Y. (2016b). Efecto de tratamientos pre-germinativos en la calidad de plantas Guapinol (Hymenaea courbaril). Nexo Revista Cientifica, Universidad Nacional de Ingeniería (UNI), Nicaragua, (ISSN 1998-8850).

García-Pérez, A. (2010). Métodos avanzados de estadística aplicada. Métodos robustos y de remuestreo. Universidad Nacional a Distancia.

IBM ${ }^{\circledR}$ Statistical SPSS ${ }^{\circledR}$. (2016). IBM ${ }^{\circledR}$ SPSS ${ }^{\circledR}$ 23.0. Retrieved from http://www.spss.com/

Instito Nicaragüense de Estudios Territoriales (INETER). (2015). Los Ecosistemas de Nicaragua y su Estrategia.

Instito Nicaragüense de Tecnología Agropecuaria (INTA). (2009). Guía tecnológica para la produción de Frijol. El autor, Segunda Ed, 1-32.

Medrano, H., y Flexas, J. (2003). Respuesta de las plantas al estrés hídrico. In La ecofisiología vegetal: una ciencia de síntesis (pp. 253-286). Retrieved from https://dialnet.unirioja.es/servlet/articulo?codigo=830199

Meriño, Y., Boudet, A., Boicet, T., Barreiro, E., Palacio, A., y Oduardo, R. (2015). Rendimiento y tolerancia a la sequía de seis variedades de frijol común (Phaseolus vulgaris L.) en condiciones de campo. Centro Agricola, 42(1), 69-74. Retrieved from http://cagricola.uclv.edu.cu/descargas/pdf/V42-Numero_1/cag10115.pdf

Mivilla, B., González, A., y Marulanda, D. (1986). Necesidades hidricas de dos variedades de frijol (Phaseolus vulgaris) en condiciones de invernadero. Acta Agronomica, 36(2), 168-176.

Monzón, D., y Monzón Paiva, D. (1992). Introducción al diseño de experimentos. Revista de La Facultad de Agronomía de La Universidad Central de Venezuela, (Alcance 34), 167 p.

Moreno, L. (2009). Respuesta de las plantas al estrés por déficit hídrico. Agronomía Colombiana, 27(2), 179-191. Retrieved from http://www.scielo.org.co/img/revistas/agc/v27n2/v27n2a06.pdf

Prado, W., Jerez, E., Nápoles, M., Sosa, A., Maceo, Y., y Cordoví, C. (2016). Respuesta de cultivares de frijol (Phaseolus vulgaris L.) a la sequía utilizando diferentes índices de selección. Cultivos Tropicales, 37(3), 79-84. https://doi. org/10.13140/RG.2.1.5181.2082

Tosquy-Valle, O. H., López-Salinas, E., Francisco-Nicolás, N., Alberto, J., y Bernardo, A. (2014). Genotipos de frijol negro opaco resistentes a sequía terminal. Revista Mexicana de Ciencias Agrícolas, 5(7), 1205-1217. Retrieved from http://www.scielo.org.mx/scielo.php?pid=S2007-09342014000700006\&script=sci_arttext\&tlng=en

Valladares, F. (2004). Estrés hídrico: ecofisiología y escala de la sequía. Ecología del bosque mediterráneo en un mundo cambiante (1st ed., pp. 163-190). Madrid, España: Ministerio de Medio Ambiente - EGRAF S.A.

Velarde, E. C., Sanchez, J., Peña, R. V., Chávez, L. T., Nolasco, A. Q., Pichardo, C., Baldomero, O. (2001). Evapotranspiracion. Hidrologia Aplicada, 19, 30. Retrieved from http://tarwi.lamolina.edu.pe/ echavarri/clase_vii_evapotranpiracion_def.pdf 